\title{
Soluble Factors Released by Endogenous Viable Cells Enhance the Antioxidant and Chemoattractive Activities of Cryopreserved Amniotic Membrane
}

\author{
Yi Duan-Arnold, ${ }^{1, *}$ Alexandra Gyurdieva, ${ }^{1}$ Amy Johnson, \\ Douglas A. Jacobstein, ${ }^{1,2}$ and Alla Danilkovitch ${ }^{1}$ \\ ${ }^{1}$ Osiris Therapeutics, Inc., Columbia, Maryland. \\ ${ }^{2}$ ThermoFisher Scientific, Inc., Portage, Michigan.
}

Objective: Regulation of oxidative stress and recruitment of key cell types are activities of human amniotic membrane (hAM) that contribute to its benefits for wound treatment. Progress in tissue preservation has led to commercialization of hAM. The majority of hAM products are devitalized with various degrees of matrix alteration. Data show the importance of hAM matrix preservation, but little is known about the advantages of retaining viable endogenous cells. In this study, we compared the antioxidant and chemoattractive properties of viable intact cryopreserved hAM (int-hAM) and devitalized cryopreserved hAM (dev-hAM) to determine the benefits of cell preservation. Approach: We evaluated the ability of int-hAM and dev-hAM to protect fibroblasts from oxidant-induced cell damage, to suppress oxidants, and to recruit fibroblasts and keratinocytes in vitro.

Results: Both the int-hAM-derived conditioned medium (CM) and the inthAM tissue rescued significantly more fibroblasts from oxidant-induced damage than dev-hAM ( $844 \%$ and $93 \%$ more, respectively). The int-hAM CM showed a $202 \%$ greater antioxidant capacity than dev-hAM. The int-hAM $\mathrm{CM}$ enhanced the recruitment of fibroblasts and normal and diseased keratinocytes to a greater extent than dev-hAM (1,555\%, 315\%, and $151 \%$ greater, respectively).

Innovation and Conclusion: Int-hAM, in which all native components are preserved, including endogenous viable cells, demonstrated a significantly greater antioxidant and fibroblast and keratinocyte chemoattractive potential compared to dev-hAM, in which viable cells are destroyed. The release of soluble factors that protect fibroblasts from oxidative injury by hAM containing viable cells is a mechanism of hAM antioxidant activity, which is a novel finding of this study.

\section{INTRODUCTION}

WOUND HEALING IS a complex multifaceted process consisting of inflammatory, proliferative, and remodeling phases. ${ }^{1}$ The inflammatory phase is the shortest among the three phases, but plays a key role in initi- ation of the healing process. During this phase, neutrophils, monocytes, and other types of blood cells migrate to the wound site and release biologically active factors, including reactive oxygen species (ROS). ${ }^{2,3}$ In moderate concentrations for a short duration,

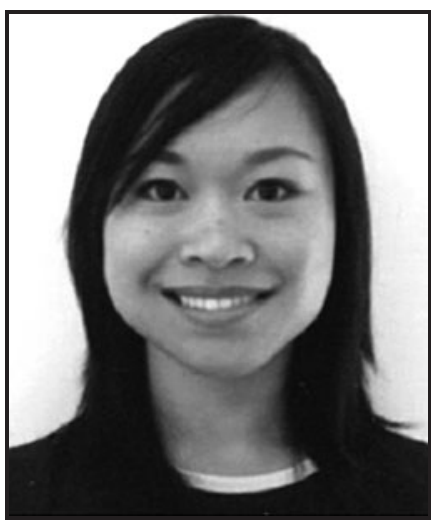

Yi Duan-Arnold, PhD

Submitted for publication January 26, 2015. Accepted in revised form March 16, 2015.

*Correspondence: Osiris Therapeutics, Inc. 7015 Albert Einstein Drive, Columbia, MD 21046 (e-mail: yarnold@osiris.com).

(c) Yi Duan-Arnold, et al. 2015; Published by Mary Ann Liebert, Inc. This Open Access article is distributed under the terms of the Creative Commons Attribution Noncommercial License (http:// creativecommons.org/licenses/by-nc/4.0/) which permits any noncommercial use, distribution, and reproduction in any medium, provided the original author(s) and the source are credited. 
ROS are important for defense against microorganisms. However, excessive ROS accumulation can lead to cell senescence and death. ${ }^{4}$ In addition, high levels of ROS increase the expression of matrix metalloproteinases that lead to destruction of extracellular matrix (ECM), further resulting in tissue damage and perpetuation of inflammation. ${ }^{5-7}$ Therefore, in chronic wounds that are characterized by prolonged inflammation, ROS must be controlled to support the transition to the next phase of wound healing.

As the inflammatory phase subsides, the wound shifts into the proliferative phase, during which granulation tissue formation, wound contraction, and re-epithelialization occur. These tissue regenerative processes require the coordinated migration of various cell types, including fibroblasts and keratinocytes, into the wound bed. ${ }^{1}$ Recruitment of these cells from the periphery to the wound bed is essential for wound healing; therefore, advanced wound care products should have this property.

Fresh human amniotic membrane (hAM) has properties that are beneficial for wound treatment. However, a short shelf life and insufficient time for screening to prevent the transmission of communicable diseases limit the use of fresh hAM. ${ }^{8}$ To ensure safety while retaining the biological activities of fresh hAM, different preservation methods have been introduced in recent years making the commercialization of hAM possible. ${ }^{9}$ In contrast to fresh hAM, the majority of hAM commercial products are devitalized (i.e., contain no viable cells) and may sustain varying degrees of damage to the structural matrix and to the pool of growth factors. ${ }^{10}$ Thus far, the effects of devitalization on the functionality of hAM are not well understood.

The goal of this study was to evaluate the antioxidant and the fibroblast and keratinocyte chemoattractive potential of viable intact cryopreserved hAM (int-hAM), which was processed by a cryopreservation method involving a controlled cooling rate allowing preservation of endogenous viable cells, and devitalized cryopreserved hAM (dev-hAM) containing no viable cells.

\section{CLINICAL PROBLEM ADDRESSED}

While fresh hAM has a long history of wound treatment, recent advances in preservation techniques have allowed hAM to become an off-the-shelf product that is readily available to physicians. However, the effects of different preservation methods on the functionality of preserved hAM largely remain to be explored. This study investigates the effect of preserving all native hAM components, including viable endogenous cells, on the ability of hAM to protect host cells from oxidant-induced damage and recruitment of host fibroblasts and keratinocytes in vitro. Both these features are desirable for products intended for the treatment of chronic wounds.

\section{MATERIALS AND METHODS}

\section{Tissue procurement and ethics statement}

Human term placentas were provided by the National Disease Research Interchange (Philadelphia, PA) and Cord Blood America, Inc. (Las Vegas, NV) from eligible donors after obtaining written informed consent.

\section{Placental tissue processing}

Placental tissues were aseptically processed in a biological safety cabinet within $36 \mathrm{~h}$ after collection. The hAM was separated from the umbilical cord and from the chorion by blunt dissection. Residual blood was removed, and the hAM was incubated in Dulbecco's modified Eagle's medium (DMEM) (GE Healthcare Life Sciences, Piscataway, NJ) containing an antibiotic cocktail of gentamicin (Fresenius Kabi, Lake Zurich, IL), vancomycin (Hospira, San Jose, CA), and Amphotericin B (Sigma-Aldrich, St. Louis, MO) for $18-48 \mathrm{~h}$ at $37^{\circ} \mathrm{C}$ and $5 \% \mathrm{CO}_{2}$ in a humidified atmosphere. Subsequently, residual antibiotics were removed by washing with Dulbecco's phosphatebuffered saline (DPBS) (Life Technologies, Carlsbad, CA), and the hAM was cut into $25 \mathrm{~cm}^{2}$ pieces.

Cryopreservation of hAM was performed by freezing hAM in a dimethyl sulfoxide (DMSO) (Mylan, Inc., Canonsburg, PA)-containing cryoprotectant solution at a controlled cooling rate, according to a proprietary process developed by Osiris Therapeutics, Inc. Tissues that were prepared solely by cryopreservation will be referred to as int-hAM in the study. Tissues prepared with cryopreservation and an additional freezing step that resulted in devitalization will be referred to as dev-hAM. Int-hAM and dev-hAM were stored at $-80^{\circ} \mathrm{C}$ for up to 3 months before their use in the experiments. Both int-hAM and dev-hAM were thawed in a room temperature water bath and rinsed with room temperature saline or DPBS before use. Placentas from multiple donors were used in this study.

\section{Generation of conditioned medium}

The conditioned medium (CM) was generated by incubating int-hAM and dev-hAM in $1 \mathrm{~mL}$ of low serum culture medium, DMEM with $1 \%$ fetal bovine serum (FBS) (Life Technologies), for 3 days at $37^{\circ} \mathrm{C}$ 
and $5 \% \mathrm{CO}_{2}$ in a humidified atmosphere. The $\mathrm{CM}$ was collected, centrifuged at $300 \mathrm{~g}$ for $5 \mathrm{~min}$ to remove cells and tissue debris, and transferred into new tubes. Samples were stored at $-80^{\circ} \mathrm{C}$ until further testing.

\section{Assessment of placental membrane cell viability}

For an assessment of the cell viability of inthAM and dev-hAM, the LIVE/DEAD ${ }^{\circledR}$ Viability/ Cytotoxicity Kit (Life Technologies) was used according to the manufacturer's instructions. Photographs were taken for both green and red channels, which were then merged using ImageJ, a Java ${ }^{\mathrm{TM}}$-based image processing program provided by the National Institute of Health (NIH, Bethesda, MD).

\section{Cell culture of fibroblasts and keratinocytes}

Human dermal fibroblasts (HDF) (Lonza, Walkersville, MD) were cultured in fibroblast growth medium (FGM-2) (Lonza) at $37^{\circ} \mathrm{C}$ and $5 \%$ $\mathrm{CO}_{2}$ in a humidified atmosphere until they reached $\sim 80 \%$ confluence and were assayed during their growth phase. Primary normal and diseased (type 2 diabetic) epidermal keratinocytes (normal human epidermal keratinocytes [NHEK] and diseased human epidermal keratinocytes [DHEK], respectively) (Lonza) were cultured in keratinocyte growth medium (KGM-2) (Lonza) at $37^{\circ} \mathrm{C}$ and $5 \%$ $\mathrm{CO}_{2}$ in a humidified atmosphere until they reached $\sim 80 \%$ confluence and were assayed during their growth phase.

\section{Fibroblast rescue from oxidant-induced cell damage by hAM in vitro}

Oxidative cell damage was induced using a modified method described by Kim et al. ${ }^{11}$ Briefly, $\mathrm{HDF}$ were plated at a density of $5.0 \times 10^{4}$ cells per well in a 24-well plate in FGM-2 and cultured for $6 \mathrm{~h}$. HDF were then serum starved in DMEM with $0.1 \%$ FBS overnight before oxidant treatment. Oxidative injury was induced by adding tert-butyl hydroperoxide (tbOOH) (Sigma-Aldrich) to HDF at a final concentration of $200 \mu \mathrm{M}$ for $3 \mathrm{~h}$. Subsequently, either medium was replaced by the CM derived from int-hAM or dev-hAM with the addition of $200 \mu \mathrm{M}$ fresh tbOOH (final concentration), or int-hAM and dev-hAM tissue samples were added into the upper chamber of a Transwell ${ }^{\circledR}$ system (Corning, Corning, NY) and cultured with the HDF for an additional $4 \mathrm{~h}$. HDF cultured with tbOOH alone or with tbOOH and $250 \mu \mathrm{M}$ ascorbic acid (Sigma-Aldrich) served as negative and positive controls, respectively. After $4 \mathrm{~h}, \mathrm{HDF}$ were fixed with $4 \%$ paraformaldehyde (USB, Cleveland, $\mathrm{OH}$ ), washed with DPBS, and stained with $10 \mu \mathrm{g} / \mathrm{mL}$ Hoechst 33342 (BD Pharmingen, San Diego, CA) for $30 \mathrm{~min}$ at $37^{\circ} \mathrm{C}$. Hoechst 33342 is a nuclear stain that binds to double-stranded DNA and emits blue fluorescence. Samples were analyzed by florescent microscopy (Nikon Eclipse TE300; Nikon, Tokyo, Japan), and representative images were taken. Total, normal, and damaged cell numbers were quantified using ImageJ (NIH). Damaged cells were identified as cells containing intensely condensed chromatin and/or fragmented nuclei. At least three independent microscopic fields from each well were used for quantification. The percent of cells rescued from oxidative damage was presented as \% recovery, which was calculated by the following formula: (normal cells/total cells) $\times 100$. The \% recovery for each sample was corrected to take baseline recovery into account using the following formula: \% recovery of sample $-\%$ recovery of negative control.

\section{Antioxidant capacity of CM derived from hAM}

The antioxidant capacity of CM derived from inthAM and dev-hAM was evaluated using an oxidant capacity kit (Sigma-Aldrich) according to the manufacturer's protocol. This assay is based on the formation of a cation radical, 2,2'-azino-bis(3ethylbenzthiazoline-6-sulfonic acid), which is green in color with an absorbance that can be detected spectrophotometrically at $405 \mathrm{~nm}$. Antioxidants suppress the production of the radical cation in a concentration-dependent manner, and the color intensity decreases proportionally. Trolox, a water-soluble vitamin $\mathrm{E}$ analog, served as a standard. The culture medium and $250 \mu \mathrm{M}$ ascorbic acid (Sigma-Aldrich) served as negative and positive controls, respectively.

\section{Cell migration assays}

The ability of hAM to stimulate the migration of HDF, NHEK, and DHEK was determined using 24-well plates and FluoroBlok ${ }^{\mathrm{TM}}$ Transwell filters with $8-\mu \mathrm{m}$ pores (BD Biosciences, Franklin Lakes, NJ). Cells were cultured as described above, and $1.0 \times 10^{5}$ cells of each type were seeded on top of the porous Transwell insert membrane and allowed to adhere overnight. Before addition of CM, cells had been starved in a low-serum medium. The CM derived from int-hAM and dev-hAM was added to the lower chambers of the Transwell. After overnight incubation, cells that had migrated through the filter were stained with calcein AM (Life Technologies) and fixed with $4 \%$ paraformaldehyde. The samples were analyzed by fluorescent microscopy (Nikon Eclipse TE300; Nikon), and representative images were taken. The FluoroBlok inserts enable 
visualization of only cells that have migrated to the bottom of the insert. The number of migrated cells was quantified by determining the percentage of the total area per field occupied by cells using ImageJ (NIH).

\section{Evaluation of cytokine and growth factor profile in CM derived from int-hAM and dev-hAM}

The presence of the following cytokines and growth factors, known to contribute to cell recruitment, in the int-hAM and dev-hAM CM was tested from three pooled donors: endothelial growth factor (EGF); vascular endothelial growth factor (VEGF); basic fibroblast growth factor (bFGF); platelet-derived growth factor (PDGF)-BB; interleukin (IL)-6; and IL-8.

The presence of IL-6, and IL-8 in the int-hAM and dev-hAM CM was investigated using a multiplex chemiluminescent array (Aushon Biosystems, Billerica, MA). For VEGF and PDGF-BB testing, $250 \mu \mathrm{L} \mathrm{CM}$ was incubated with $250 \mu \mathrm{L} 8 \mathrm{M}$ guanidine $\mathrm{HCl}$ (Thermo Fisher Scientific, Waltham, MA) containing protease inhibitor (Roche, Basel, Switzerland) at $4^{\circ} \mathrm{C}$ overnight with rotation. Samples were centrifuged at $300 \mathrm{~g}$ for $10 \mathrm{~min}$ at $4^{\circ} \mathrm{C}$ and then desalted using Zeba Desalting Columns (Thermo Fisher Scientific) as per the manufacturer's instructions. Desalted samples were collected and stored at $-80^{\circ} \mathrm{C}$ until further testing by enzymelinked immunosorbent assay (ELISA) kits (R\&D Systems, Minneapolis, MN) as per the manufacturer's instructions. The guanidine $\mathrm{HCl}$ treatment of samples before measurement of VEGF and PDGF$\mathrm{BB}$ was performed to eliminate factors that interfere with VEGF and PDGF-BB detection by ELISA. The presence of EGF and bFGF in the CM was also tested by ELISA as per the manufacturer's instructions (R\&D Systems).

\section{Statistical analysis}

Data are presented as mean \pm standard deviation (SD) for one representative experiment out of three with each experiment consisting of three biological replicates. Student's $t$-test was used for statistical analysis comparing int-hAM and devhAM, and $p<0.05$ was considered significant.

\section{RESULTS}

\section{Cellular viability is maintained in int-hAM but not dev-hAM}

To confirm the presence of viable cells in inthAM and the absence of viable cells in dev-hAM, the LIVE/DEAD viability/cytotoxicity assay was performed. The majority of the endogenous cells in both the epithelial and stromal layers of int-hAM remained viable after thawing (Fig. 1A). In comparison, dev-hAM contained only dead cells (Fig. 1B).

\section{Int-hAM protects fibroblasts from oxidant-induced damage in vitro}

To investigate the ability of hAM to protect cells from oxidant-induced damage, an in vitro tbOOHinduced HDF oxidative injury model was used. A confluent layer of $\mathrm{HDF}$ was treated with tbOOH in the absence or presence of CM or tissue samples derived from int-hAM or dev-hAM. Cell damage was determined by Hoechst staining. As depicted in Figure 2A, cells treated with tbOOH alone (negative control) exhibited morphological characteristics of apoptosis, including cell shrinkage, chromatin condensation, and nuclear fragmentation. In contrast, when HDF were treated with both tbOOH and ascorbic acid, a known antioxidant, $76 \% \pm 1 \%$ of cells recovered from oxidative stress (Fig. 2A, B, positive control). When int-hAM tissue samples were added to the oxidant-damaged $\mathrm{HDF}$, $74 \% \pm 7 \%$ of cells were rescued (Fig. $2 \mathrm{~B}$ ), which is comparable to the effect observed with the positive control. In contrast, only $38 \% \pm 13 \%$ of cells were rescued by the dev-hAM tissue (Fig. 2B). To examine soluble antioxidants, the CM was used. The $\mathrm{CM}$ derived from int-hAM rescued $70 \% \pm 6 \%$ of damaged cells, whereas CM derived from dev-hAM had a negligible effect ( $7 \% \pm 8 \%$ ) (Fig. 2A, B).

\section{Int-hAM possesses higher antioxidant capacity than dev-hAM}

The antioxidant capacity of CM derived from inthAM and dev-hAM was measured by its ability to suppress the formation of free radicals. Trolox, a water-soluble vitamin E analog, served as a standard, and results are presented as the equivalent of $\mu \mathrm{M}$ of Trolox. The CM derived from int-hAM had a high antioxidant capacity equivalent to $322 \pm 35 \mu \mathrm{M}$ of Trolox, which was comparable with the capacity of $250 \mu \mathrm{M}$ ascorbic acid (positive control) (Fig. 2C). In contrast, the CM derived from dev-hAM had an activity equivalent to only $107 \pm 5 \mu \mathrm{M}$ of Trolox (Fig. 2C).

\section{Effect of int-hAM and dev-hAM on fibroblast migration in vitro}

To evaluate the chemoattractive effect of hAM on fibroblasts, a Transwell migration assay was used. HDF were seeded on top of a porous Transwell membrane, and the cells that migrated through the membrane toward the hAM-derived CM were quantified. The CM derived from int-hAM induced migration of HDF comparable to the positive control (FGM-2 containing chemoattractive growth 
A


B
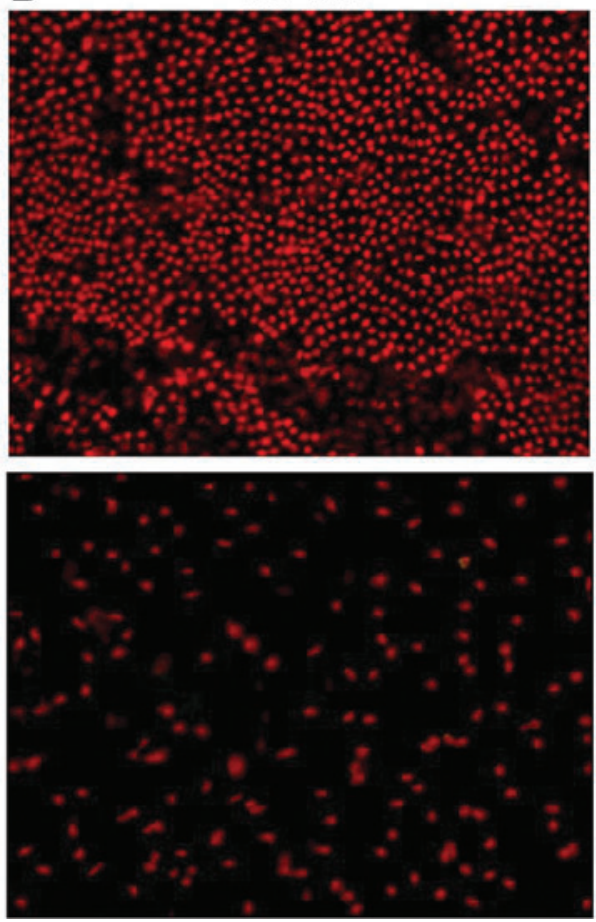

Figure 1. Live and dead endogenous cell staining of hAMs. Live and dead cells in epithelial and stromal layers of viable intact cryopreserved human amniotic membrane (int-hAM) post-thaw (A) and devitalized cryopreserved human amniotic membrane (dev-hAM) post-thaw (B) were visualized microscopically using the LIVE/DEAD ${ }^{\circledR}$ Viability/Cytotoxicity Kit. Live cells stained green with calcein AM. Dead cells stained red with ethidium homodimer-1. All images were taken at $10 \times$ magnification.

factors): migrated cells covered $22 \% \pm 0.3 \%$ and $32 \% \pm 5 \%$ total area/field, respectively (Fig. 3). In contrast, migrated cells covered only $1 \% \pm 2 \%$ total area/field with the CM derived from dev-hAM.

\section{Effect of int-hAM and dev-hAM on normal and diseased keratinocyte migration in vitro}

The migration of both NHEK and DHEK was also examined, respectively. DHEK are harvested from patients with type 2 diabetes. The CM derived from int-hAM enhanced the migration of both cell types relative to dev-hAM: migrated NHEK covered $11 \% \pm 2 \%$ versus $3 \% \pm 2 \%$ total area/field, respectively; and migrated DHEK covered $12 \% \pm 1 \%$ versus $5 \% \pm 1 \%$ of total area/field, respectively (Figs. 4 and 5). Cell migration induced by the inthAM-derived CM is comparable to cell migration induced by positive control, where the migrated NHEK and DHEK covered $14 \% \pm 1 \%$ and $11 \% \pm 2 \%$ of total area/field, respectively.

\section{Presence of chemoattractive cytokines and growth factors in int-hAM- and dev-hAM-derived $\mathrm{CM}$}

The CM derived from int-hAM and dev-hAM was analyzed to determine if the enhanced cell recruitment by int-hAM is due to higher concentra- tions of chemoattractive cytokines and growth factors. Our data demonstrated that the dev-hAM CM contained higher levels of EGF while int-hAM contained higher levels of VEGF, IL-6, bFGF, PDGF-BB, and IL-8 (Fig. 6).

\section{DISCUSSION}

The primary objective of this study was to evaluate the significance of preserving tissueresident cells, along with ECM and growth factors, on the antioxidant and cell recruitment potential of hAM. The results demonstrated that compared to dev-hAM, which contains no viable cells, int-hAM retains viable endogenous cells. Int-hAM better protected fibroblasts from oxidant-induced damage, had increased antioxidant capacity, and promoted fibroblast and keratinocyte migration to a greater extent.

ROS play an important role in the inflammatory phase of wound healing, and in acute wounds, ROS levels are tightly controlled by endogenous antioxidant defense systems. ${ }^{3}$ However, prolonged exposure to ROS and decreased levels of antioxidants associated with chronic wounds result in increased oxidative stress; this has been demonstrated in 

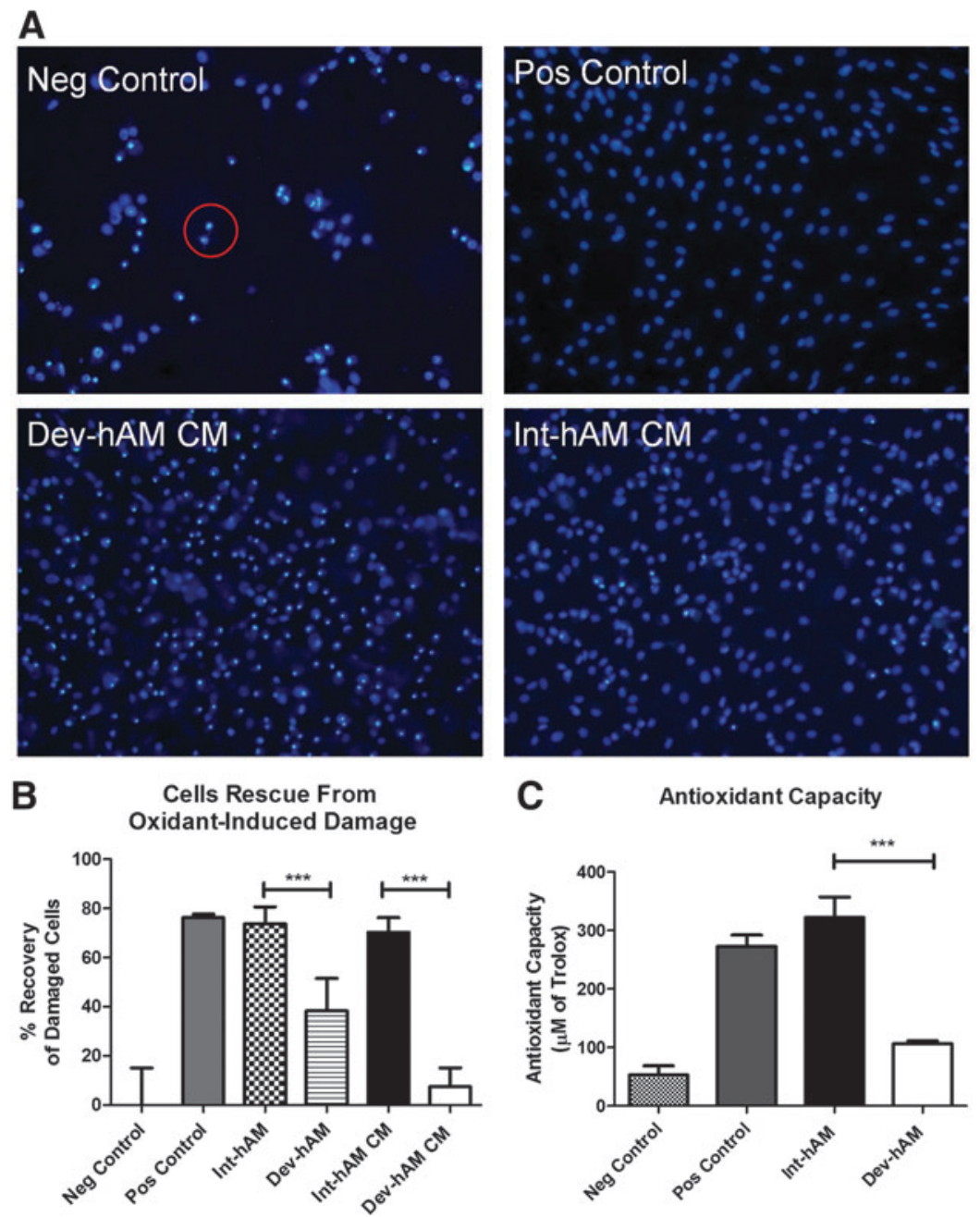

Figure 2. Antioxidant capacity of hAM and effects of hAM on oxidant-induced damage of HDF. Oxidant damage in HDF was induced by addition of $200 \mu \mathrm{M}$ of tbOOH. To evaluate if hAM can rescue cells from oxidant-induced damage, viable intact cryopreserved human amniotic membrane (int-hAM) and devitalized cryopreserved human amniotic membrane (dev-hAM) tissue pieces or CM was added to HDF cultures. Cell damage was determined by Hoechst staining. Damaged cells were identified by condensed chromatin (red circle). The number of total, normal, and damaged cells was quantified using Image JM (NIH). Results are presented as \% recovery, which was calculated by the following formula: (normal cells / total cells) $\times 100$. The $\%$ recovery for each sample was corrected using the following formula: \% recovery of sample - \% recovery of negative (Neg) control. HDF cultured in a medium with tbOOH with or without ascorbic acid served as positive (Pos) and negative controls, respectively. Representative microscopic images of HDF cultures at $10 \times$ magnification are shown in (A). Results of the quantitative analysis are shown in (B). The antioxidant capacity of CM derived from int-hAM and dev-hAM was tested by their ability to suppress the formation of ROS in the presence of hydrogen peroxide using an antioxidant assay kit. The antioxidant capacity of int-hAM and dev-hAM is presented as $\mu \mathrm{M}$ of Trolox. Trolox, a water-soluble vitamin $\mathrm{E}$ analog, served as a standard. Medium with and without ascorbic acid, a known antioxidant, served as positive (Pos) and negative (Neg) controls, respectively. Results are shown in (C). Data are presented as mean \pm SD of one representative experiment out of three. Student's $t$-test was used for statistical analysis comparing int-hAM and dev-hAM. ${ }^{* * *} p<0.001$. CM, conditioned medium; HDF, human dermal fibroblasts; ROS, reactive oxygen species; tbOOH, tert-butyl hydroperoxide.

animal studies. ${ }^{12,13}$ In a diabetic mouse model reported by Dhall et al., a high level of oxidative stress resulted in the formation of chronic wounds that were then reversed by treatment with antioxidants. ${ }^{12}$ It has been recommended that exogenous antioxidants should be included in the treatment regimen for chronic wounds to control ROS imbalance.

The antioxidant properties of hAM have largely remained unexplored. One recent study by Lockington et al. examined the antioxidant potential of
hAM and found that it can inactivate hydrogen peroxide, a common oxidant. ${ }^{13}$ In our study, we utilized a tbOOH-induced $\mathrm{HDF}$ oxidative injury model to evaluate the antioxidant potential of int$\mathrm{hAM}$ and to compare it to dev-hAM. The potential of both the hAM tissue samples and of hAMderived CM was studied to elucidate the contribution of the tissue matrix and to determine if released soluble antioxidant factors were present. Int-hAM tissue samples rescued $74 \%$ of HDF, but only $38 \%$ of cells were rescued by dev-hAM tissue (Fig. 2). 
A
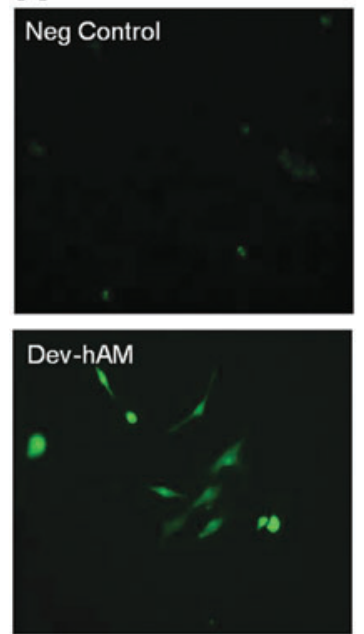

B
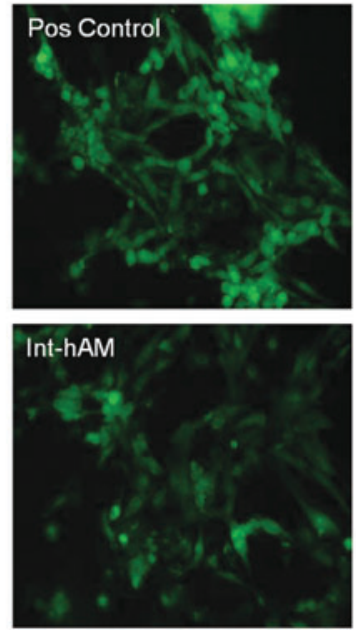

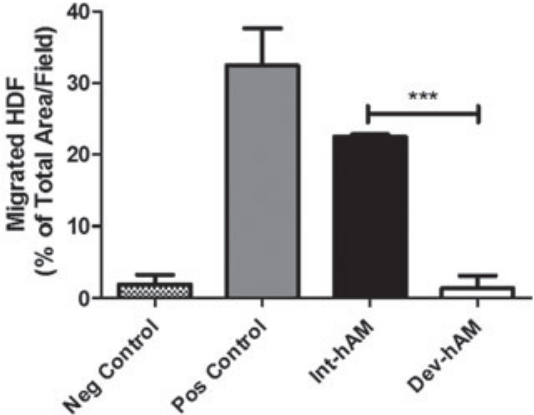

Figure 3. Effect of hAMs on HDF migration. The migration of HDF induced by CM derived from viable intact cryopreserved human amniotic membrane (inthAM) and devitalized cryopreserved human amniotic membrane (dev-hAM) was examined. HDF were incubated with CM overnight, and migrated cells were stained with calcein AM and visualized microscopically. The area of migrated HDF was quantified using ImageJ (NIH), and results are presented as \% of Total Area/Field. Base medium was used as a negative ( $\mathrm{Neg}$ ) control, and FGM-2 was used as a positive (Pos) control. Images of migrated HDF at $10 \times$ magnification for one representative experiment are shown in (A). Results of quantitative analysis are shown in (B). Data are presented as mean \pm SD of three independent microscopic fields from one representative experiment. Student's $t$-test was used for statistical analysis comparing int-hAM and dev-hAM. ${ }^{* * *} p<0.001$. CM, conditioned medium; FGM-2, fibroblast growth medium; HDF, human dermal fibroblasts.

The CM derived from int-hAM rescued $70 \%$ of damaged HDF, which is similar to the effect observed with the use of int-hAM tissue (Fig. 2). In contrast, the CM derived from dev-hAM had a negligible effect (7\%), which was significantly lower than the effect of dev-hAM tissue (38\%). These results indicate that hAM endogenous viable cells are important for the release of soluble factors that protect fibroblasts from oxidative injury.

Lockington et al. have attributed the antioxidant potential of devitalized hAM to high-molecularweight hyaluronic acid (HA), which has been shown to decrease oxidative DNA damage in corneal epithelial cells. ${ }^{13,14}$ We did not test for high-molecular-
A
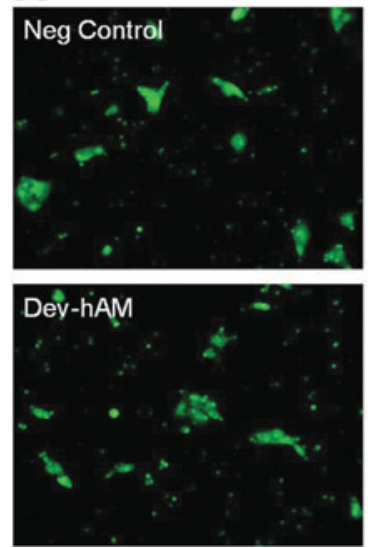
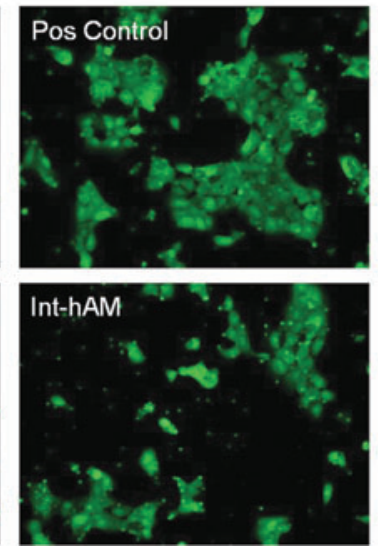

B

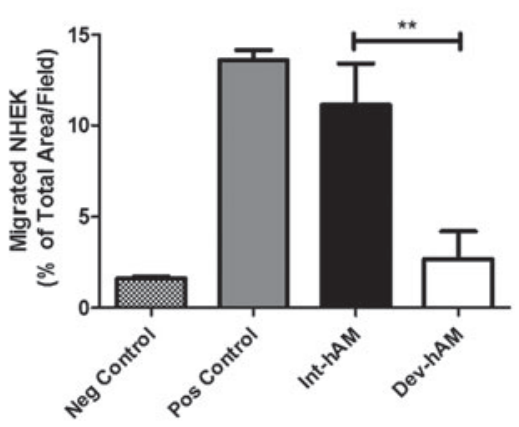

Figure 4. Effect of hAMs on NHEK migration. The migration of NHEK induced by CM derived from viable intact cryopreserved human amniotic membrane (inthAM) and devitalized cryopreserved human amniotic membrane (dev-hAM) was examined. NHEK were incubated with CM overnight, and migrated cells were stained with calcein AM and visualized microscopically. The area of migrated NHEK was quantified using ImageJ (NIH), and results are presented as \% of Total Area/Field. Base medium was used as a negative (Neg) control, and KGM-2 was used as a positive (Pos) control. Images of migrated NHEK at $10 \times$ magnification for one representative experiment are shown in (A). Results of quantitative analysis are shown in (B). Data are presented as mean \pm SD of three independent microscopic fields from one representative experiment. Student's $t$-test was used for statistical analysis comparing int-hAM and dev-hAM. ${ }^{* *} p<0.01$. CM, conditioned medium; KGM-2, keratinocyte growth medium; NHEK, normal human epidermal keratinocytes. 
A
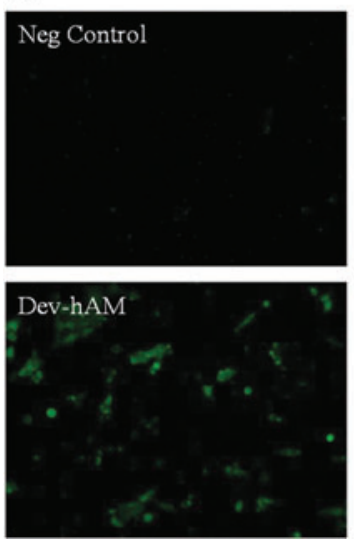
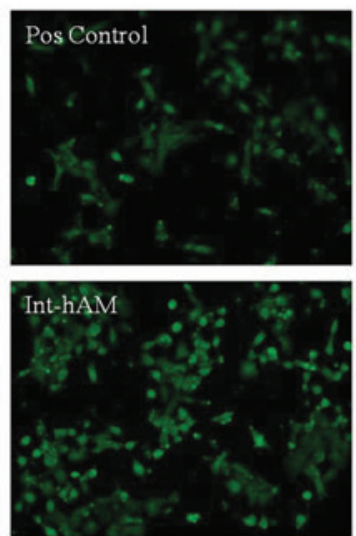

B

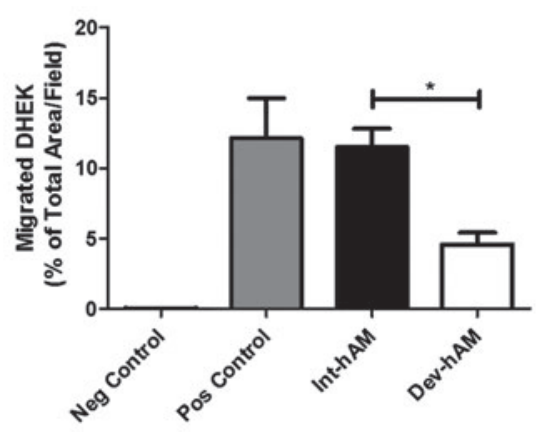

Figure 5. Effect of hAMs on DHEK migration. The migration of DHEK obtained from patients with type 2 diabetes was induced by CM derived from viable intact cryopreserved human amniotic membrane (int-hAM) and devitalized cryopreserved human amniotic membrane (dev-hAM). DHEK were incubated with CM overnight, and migrated cells were stained with calcein AM and visualized microscopically. The area of migrated DHEK was quantified using ImageJ (NIH), and results are presented as \% of Total Area/Field. Base medium was used as a negative (Neg) control, and KGM-2 was used as a positive (Pos) control. Images of migrated DHEK at $10 \times$ magnification for one representative experiment are shown in (A). Results of quantitative analysis are shown in (B). Data are presented as mean $\pm S D$ of three independent microscopic fields in one representative experiment. Student's $t$-test was used for statistical analysis comparing int-hAM and dev-hAM. ${ }^{*} p<0.05$. CM, conditioned medium; DHEK, diseased human epidermal keratinocytes; KGM-2, keratinocyte growth medium.

weight HA in this study. However, it has been reported that devitalized cryopreserved amnion retains high-molecular-weight HA. ${ }^{15}$ We do not know if int-hAM and dev-hAM retained significantly different levels of high-molecular-weight HA, which could contribute to the difference observed between int-hAM tissue and dev-hAM tissue. In addition, this difference between int-hAM and devhAM could also involve other antioxidants not yet identified in hAM that mediate the int-hAM pro-

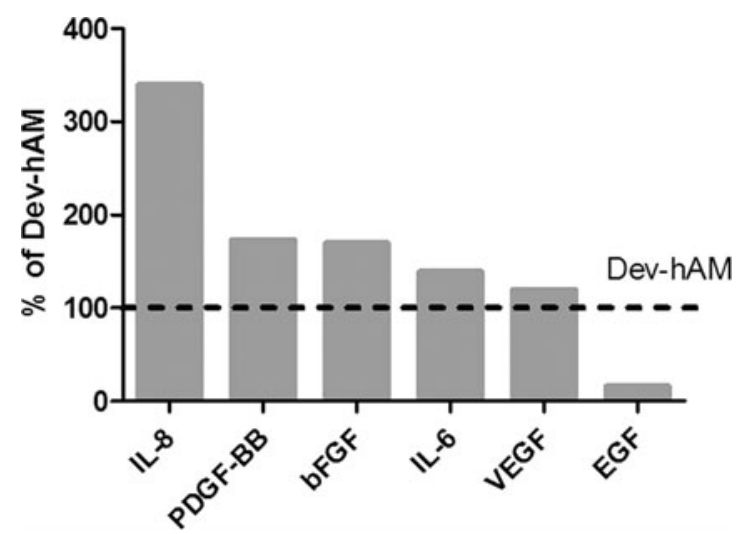

Figure 6. Chemoattractive cytokines and growth factors in hAMs. Chemoattractive cytokines and growth factors in 3-day CM derived from viable intact cryopreserved human amniotic membrane (int-hAM) and devitalized cryopreserved human amniotic membrane (dev-hAM) were measured using single factor or multiplex immunoassays. Data are presented as levels of growth factors or cytokines in int-hAM as a percentage of those in devhAM. Levels in dev-hAM were considered $100 \%$. CM, conditioned medium. tective effect and are absent or present at low levels in dev-hAM.

We found a large difference between the protective effects of int-hAM CM and dev-hAM CM on oxidantdamaged cells indicating that viable hAM can release soluble antioxidants that suppress ROS. We confirmed this hypothesis and found that the int-hAM CM has a $202 \%$ greater antioxidant capacity compared with dev-hAM CM. In summary, these data indicate that (1) the antioxidant effect of int-hAM and dev-hAM tissues is mediated by matrix components, one of which might be high-molecular-weight HA and (2) int-hAM releases additional soluble antioxidants, which represents another mechanism of hAM antioxidant activity that is lost in dev-hAM.

Some of these soluble factors released by inthAM may include ROS-scavenging enzymes such as superoxide dismutase (SOD), catalase, and glutathione peroxidase $(\mathrm{GPx}){ }^{16}$ Low-molecularweight antioxidants such as uric acid may also be involved. ${ }^{17} \mathrm{Kim}$ et al. reported that $\mathrm{CM}$ from adipose-derived mesenchymal stem cells (ASC) contains soluble factors that protect fibroblasts from oxidative stress through (1) the scavenging of ROS by antioxidant molecules such as SODs and (2) the release of growth factors such as insulin-like growth factor and PDGF that activate cell membrane receptors and ultimately increase SOD and GPx activity in fibroblasts. ${ }^{11,18}$ Mesenchymal stem cells (MSC) are present in hAM. ${ }^{19}$ Potentially, viable MSCs in int-hAM may mediate cell protection from oxidative stress through similar mechanisms. 
In these experiments by Kim et al., cells were only protected if they were pretreated with ASC-CM before exposure to an oxidant. The protective effect diminished when cells were exposed to the oxidant and ASC-CM simultaneously. ${ }^{11}$ In contrast, our results demonstrate that int-hAM-derived CM is able to protect HDF from ongoing oxidative stress, which is a more relevant condition to the chronic wound microenvironment. This

\section{KEY FINDINGS}

- Preservation of viable endogenous cells in cryopreserved hAM augments its antioxidant and its fibroblast and keratinocyte chemoattractive activities.

- The antioxidant activity of hAM is mediated by two mechanisms: factors present in the structural matrix and release of soluble antioxidants.

- Viable hAM releases soluble antioxidants. Devitalization of hAM results in the loss of antioxidant activity mediated by the release of soluble factors. indicates that int-hAM-derived CM con-

tains several factors that mediate the int-hAM antioxidant activity through different mechanisms. The identification of the antioxidant factors released from int-hAM and their mechanisms of cell protection from oxidant-induced injury remain to be answered.

Upon resolution of chronic inflammation and transition of a wound from the inflammatory to the proliferative phase of healing, the cascade of collagen deposition, granulation tissue formation, and re-epithelialization requires the recruitment of cells to the wound area. ${ }^{1}$ Studies have shown that hAM-derived cells have the ability to secrete chemoattractive factors that stimulate the migration of keratinocytes and fibroblasts. ${ }^{20,21}$ Therefore, we hypothesized that int-hAM, in which all components of fresh hAM are preserved, could have a stronger effect on the migration of cells. Our results demonstrated that int-hAM CM recruited significantly more fibroblasts compared to devhAM (Fig. 3). Although we observed a statistically significant difference in the migration of normal and type 2 diabetic patient-derived keratinocytes (Figs. 4 and 5) between int-hAM and dev-hAM, the effect was not as strong as the effect of int-hAM and dev-hAM on fibroblast migration (Fig. 3).

A number of different cytokines and growth factors are known to induce fibroblast and keratinocyte migration. Growth factors such as EGF, VEGF, bFGF, and PDGF-BB can contribute to the recruitment of fibroblasts and keratinocytes. ${ }^{22}$ In addition, cytokines such as IL-6 and IL-8, known to be produced by MSCs, have been shown to be potentially involved in the stimulation of cell $\mathrm{mi}-$ gration. ${ }^{23}$ We found these cytokines and growth factors present in both CM derived from int-hAM and dev-hAM (Fig. 6). However, int-hAM CM overall contained a higher level of tested growth factors known to be fibroblast chemoattractants, which can be linked to the observed effect of inthAM on fibroblast migration. On the other hand, dev-hAM contained more EGF, which is one of the key growth factors for keratinocyte migration.
However, the higher level of EGF did not result in greater migration of keratinocytes, suggesting that the EGF alone is not sufficient to trigger keratinocyte migration.

In summary, the results of this study bring to light considerable differences in the magnitude of the antioxidant and chemoattractive effects of inthAM and dev-hAM. Int-hAM has a higher antioxidant potential in comparison to dev-hAM in that it protects significantly more fibroblasts from oxidative damage and suppresses more ROS. Due to soluble factors released, int-hAM recruits more fibroblasts and keratinocytes. Taken together, these data indicate that preservation of all tissue components native to $\mathrm{hAM}$ enhances its potential to protect cells from oxidative damage and to stimulate cell migration, which are essential for wound healing. One limitation of the current study is that all data were obtained in vitro. However, the results of this study suggest the merits of future research to explore whether these protective and stimulating effects of int-hAM also occur in vivo.

\section{INNOVATION}

Management of chronic wounds requires controlling ROS and stimulating the migration of cells to the wound site. In this study, we describe a cryopreserved hAM that retains all the native structural and cellular components. This correlates with a high antioxidant capacity and enhanced cell recruitment compared to dev-hAM, containing no viable cells. A new finding of this study is that viable hAM releases soluble antioxidants, a mechanism of hAM antioxidant activity that is diminished upon hAM devitalization.

\section{ACKNOWLEDGMENTS AND FUNDING SOURCES}

The authors would like thank Dr. Thomas Uveges and Dr. Malathi Sathyamoorthy for their valuable comments in reviewing the article. This research was supported by Osiris Therapeutics, Inc. 


\section{AUTHOR DISCLOSURE AND GHOSTWRITING}

All listed authors are employees of Osiris Therapeutics, Inc. This article was not written by any writer other than the authors.

\section{ABOUT THE AUTHORS}

Yi Duan-Arnold, $\mathbf{P h D}$, is currently a senior scientist at Osiris Therapeutics, Inc. (OTI). She obtained her $\mathrm{PhD}$ in Biomedical Engineering from the City College of New York followed by postdoctoral training at the Yale Medical School. After that, she worked at the Laboratory for Stem Cells and Tissue Engineering at Columbia University on cardiac tissue engineering. Alexandra Gyurdieva, MS, is a scientist at OTI. Amy Johnson, BS, is a senior research associate at OTI. Douglas A. Jacobstein, MD, was the senior medical director at OTI when this research was conducted. Currently, he holds a position at Thermo Fisher Scientific, Inc. Alla Danilkovitch, $\mathbf{P h D}$, is currently the Vice President of Research and Development at OTI. Dr. Danilkovitch has over 25 years of broad biomedical research experience, including stem cell biology, immunology, and cancer research. Dr. Danilkovitch earned a PhD degree in cell biology.

\section{REFERENCES}

1. Clark R. Overview and general considerations of wound repair. In: Clark RAF, Henson PM, eds. The Molecular and Cellular Biology of Wound Repair. New York: Springer, 1988:3-33.

2. Babior BM. Oxygen-dependent microbial killing by phagocytes (first of two parts). N Engl J Med 1978;298:659-668.

3. Sen CK. The general case for redox control of wound repair. Wound Repair Regen 2003;11:431-438.

4. Sies H. Oxidative stress: from basic research to clinical application. Am J Med 1991;91:31S-38S.

5. Buhimschi IA, Kramer WB, Buhimschi CS Thompson LP, Weiner CP. Reduction-oxidation (redox) state regulation of matrix metalloproteinase activity in human fetal membranes. Am J Obstet Gynecol 2000;182:458-464.

6. Bjermer L, Eklund A, Blaschke E. Bronchoalveolar lavage fibronectin in patients with sarcoidosis: correlation to hyaluronan and disease activity. Eur Respir J 1991;4:965-971.

7. Liu RM. Oxidative stress, plasminogen activator inhibitor 1, and lung fibrosis. Antioxid Redox Signal 2008;10:303-319.

8. Adds PJ, Hunt CJ, Dart JK. Amniotic membrane grafts, "fresh" or frozen? A clinical and in vitro comparison. Br J Ophthalmol 2001;85:905-907.

9. Amniox Medical, Inc. CRYOTEK process. http:// amnioxmedical.com/CRYOTEK-process.html (accessed January 19, 2015)

10. Cooke M, Tan EK, Mandrycky C, He H, O'Connell J, Tseng SC. Comparison of cryopreserved amniotic membrane and umbilical cord tissue with dehydrated amniotic membrane/chorion tissue. J Wound Care 2014;23:465-474, 476.

11. Kim WS, Park BS, Kim HK, et al. Evidence supporting antioxidant action of adipose-derived stem cells: protection of human dermal fibroblasts from oxidative stress. J Dermatol Sci 2008;49: 133-142.
12. Dhall S, Do DC, Garcia M, et al. Generating and reversing chronic wounds in diabetic mice by manipulating wound redox parameters. Journal of Diabetes Research. 2014;2014:562625. [Epub doi: 10.1155/2014/562625].

13. Lockington D, Agarwal P, Young D, Caslake M, Ramaesh K. Antioxidant properties of amniotic membrane: novel observations from a pilot study. Can J Ophthalmol 2014;49:426-430.

14. Wu H, Zhang H, Wang C, et al. Genoprotective effect of hyaluronic acid against benzalkonium chloride-induced DNA damage in human corneal epithelial cells. Mol Vis 2011;17:3364-3370.

15. Tan EK, Cooke M, Mandrycky C, et al. Structural and biological comparison of cryopreserved and fresh amniotic membrane tissues. J Biomater Tissue Eng 2014:4:379-388

16. Steiling H, Munz B, Werner S, Brauchle M. Different types of ROS-scavenging enzymes are expressed during cutaneous wound repair. Exp Cell Res 1999:247:484-494.

17. Schafer $M$, Werner $S$. Oxidative stress in normal and impaired wound repair. Pharmacol Res 2008:58:165-171

18. Kim WS, Park BS, Sung JH. The wound-healing and antioxidant effects of adipose-derived stem cells. Expert Opin Biol Ther 2009;9:879-887.

19. Parolini O, Alviano F, Bagnara GP, et al. Concise review: isolation and characterization of cells from human term placenta: outcome of the first international Workshop on Placenta Derived Stem Cells. Stem Cells 2008:26:300-311.

20. Uberti MG, Pierpont YN, Ko F, et al. Amnion-derived cellular cytokine solution (ACCS) promotes migration of keratinocytes and fibroblasts. Ann Plast Surg 2010;64:632-635.

21. Liu X, Wang Z, Wang R, et al. Direct comparison of the potency of human mesenchymal stem cells derived from amnion tissue, bone marrow and adipose tissue at inducing dermal fibroblast responses to cutaneous wounds. Int J Mol Med 2013:31:407-415.

22. Barrientos S, Stojadinovic O, Golinko MS, Brem H Tomic-Canic M. Growth factors and cytokines in wound healing. Wound Repair Regen 2008:16 585-601.

23. Walter MN, Wright KT, Fuller HR, MacNeil $S$, Johnson WE. Mesenchymal stem cell-conditioned medium accelerates skin wound healing: an in vitro study of fibroblast and keratinocyte scratch assays. Exp Cell Res 2010;316:1271-1281.

\section{Abbreviations and Acronyms}

bFGF $=$ basic fibroblast growth factor

$\mathrm{CM}=$ conditioned medium

Dev-hAM = devitalized cryopreserved human amniotic membrane

DHEK $=$ diseased human epidermal keratinocytes

DMEM = Dulbecco's modified Eagle's medium DPBS $=$ Dulbecco's phosphate-buffered saline

$\mathrm{ECM}=$ extracellular matrix

$\mathrm{EGF}=$ epidermal growth factor

$F B S=$ fetal bovine serum

$\mathrm{FGM}=$ fibroblast growth medium

$\mathrm{GPx}=$ glutathione peroxidase

$\mathrm{HA}=$ hyaluronic acid

hAM = human amniotic membrane

$\mathrm{HDF}=$ human dermal fibroblasts

$\mathrm{IL}=$ interleukin

Int-hAM = viable intact cryopreserved human amniotic membrane

$\mathrm{KGM}-2=$ keratinocyte growth medium

$\mathrm{MSC}=$ mesenchymal stem cell

NHEK $=$ normal human epidermal keratinocytes

$\mathrm{PDGF}=$ platelet-derived growth factor

$\mathrm{ROS}=$ reactive oxidative species

$\mathrm{SOD}=$ superoxide dismutase

tbOOH = tert-butyl hydroperoxide

$\mathrm{VEGF}=$ vascular endothelial growth factor 